\title{
Update on benefit of immunotherapy and targeted therapy in melanoma: the changing landscape
}

This article was published in the following Dove Press journal:

Cancer Management and Research

20 June 2014

Number of times this article has been viewed

\section{Neeharika Srivastava \\ David McDermott}

Department of Hematology/Oncology, Beth Israel Deaconess Medical Center, Boston, MA, USA
Correspondence: David McDermott Department of Hematology/Oncology, Beth Israel Deaconess Medical Center, 375 Longwood Ave, MASCO 428, Boston, MA 02215, USA

Tel + I 6176329270

Fax + I 6176329260

Email dmcdermo@bidmc.harvard.edu
Abstract: Malignant melanoma is on the rise. There have been recent advances in targeted agents and immunotherapies that have improved the management and treatment of patients with advanced melanoma. This review discusses the clinical efficacy and unique side effects of targeted immunotherapy and the role of predictive biomarkers in better selection of patients who would derive most benefit from specific treatments. Additionally, this review addresses concerns about the best sequencing algorithms for the currently available targeted agents. By thoroughly and extensively researching through PubMed and the American Society of Clinical Oncology, 69 published articles and abstracts were identified as addressing topics related to malignant melanoma and immunotherapy. The research was divided into subcategories discussing cytokine-based therapy, immunotherapy, molecularly targeted agents, other novel targeted agents, and combination regimens for malignant melanoma. New immune checkpoint inhibitors and targeted agents are able to improve immune-mediated regulatory effects against tumors and, specifically in advanced melanoma, are associated with improvement in overall survival. These new agents have distinct side effects that are often controlled and reversed with dose reductions and/or use of corticosteroids. Currently, there are clinical trials underway to assess the role of combination therapy, whereas other trials are focusing on devising algorithms to delineate how best to sequentially administer these drugs. Although there has been tremendous progress in the management of advanced melanoma with immunotherapy and targeted agents, there is still much to be learned about clinically useful predictive biomarkers and combination therapies as well as how to administer these agents safely.

Keywords: melanoma, immunotherapy, ipilimumab, vemurafenib, PD-1, PD-L1

\section{Introduction}

The incidence of malignant melanoma is on the rise, with over 76,250 new cases and approximately 9,000 deaths in 2012. ${ }^{1}$ The number of melanoma cases in young people (aged 18-39 years) is rapidly increasing. ${ }^{2}$ Since melanoma affects younger patients more than most other solid tumors, the average number of years of potential life lost is 15 years. ${ }^{3}$ Advances in systemic therapies have improved survival for patients with advanced melanoma; however, the 5-year survival rate remains poor. ${ }^{4}$

While cytokine-based immunotherapy remains an essential facet of the treatment of advanced melanoma in stage III disease in the adjuvant setting and in metastatic melanoma, the development of targeted therapies such as BRAF kinase inhibitors and anti-cytotoxic T-lymphocyte antigen-4 (CTLA-4) antibodies has improved the care of patients with advanced melanoma. This review discusses the clinical efficacy and unique side effects of current and future targeted immunotherapy strategies (eg, PD-1/PD-L1 antibodies). 
In addition, much work has been done to identify predictive markers to better select patients who would derive benefit from these specific therapies. Further, there has been a heightened interest in studying combination therapies and devising algorithms to determine the best sequence with which to administer these targeted agents.

\section{Cytokine-based immune therapy}

Historically, cytokine-based immunotherapy has played a major role in the management of melanoma. Recombinant interferon- $\alpha 2 b$ (IFN) has antitumor activity in melanoma, both as a single agent and in combination with chemotherapy. ${ }^{5}$ The US Food and Drug Administration (FDA) approved IFN for use in the adjuvant setting for patients with stage IIb or III disease based on study E1684, which demonstrated prolongation of both disease-free survival and overall survival in these patients. ${ }^{6,7}$ Subsequent studies of high-dose IFN in the adjuvant setting have shown statistically significant improvement in relapse-free survival. However, the data pertaining to overall survival have not been so persuasive. Although single-agent IFN has an objective response rate of $15 \%$, which increases to as high as $50 \%$ in combination with chemotherapy, fewer than $10 \%$ of treated patients experience a durable complete remission; the average response rate ranges from 6 to 9 months, and no benefit in overall survival has been demonstrated. ${ }^{5}$ Studies in patients with stage IV melanoma has not demonstrated a role for IFN in the metastatic setting. The clinical toxicities associated with IFN, specifically grade $3 / 4$ myelosuppression $(77.5 \%$ ), grade $3 / 4$ hepatotoxicity (65\%), grade $3 / 4$ neurotoxicity $(17.5 \%)$, and mild renal toxicity, greatly limit its use in patients. ${ }^{8}$ Although high-dose IFN is used in the adjuvant setting, there is still a need for better therapeutic options.

Investigators have attempted to identify predictive biomarkers for selecting patients who would benefit from adjuvant IFN. Retrospective data suggest that patients with ulcerated primary melanomas preferentially benefit from IFN therapy, with improvement in disease-free survival (odds ratio $0.51, P=0.0053) .{ }^{9}$ This was demonstrated again in a metaanalysis of Phase III data from the European Organization for Research and Treatment of Cancer (EORTC) 18952 and EORTC 18991, in which both tumor stage and ulceration were considered to be predictive factors for the efficacy of adjuvant IFN. ${ }^{10}$ The appearance of autoantibodies or clinical manifestations of autoimmunity during therapy may predict a positive response to IFN, with improvements in relapse-free survival and overall survival in patients with stage IIB, stage IIC, or stage III melanoma. ${ }^{11}$ Gogas et al ${ }^{11}$ studied single nucleotide polymorphisms of CTLA-4 associated with autoimmune disease as a predictive marker for response to IFN, but none correlated with overall survival. ${ }^{12}$ Additional investigation of predictive biomarkers for IFN is clearly needed.

In 1998, the FDA approved high-dose interleukin (IL)-2 for use in patients with metastatic melanoma. ${ }^{13}$ The overall objective response rate of high-dose IL-2 monotherapy in metastatic melanoma was only $16 \%$; however, as is true for IFN, a small group of patients (approximately 4\%) achieved a durable complete remission. ${ }^{13}$ The clinical toxicities of IL-2 therapy can lead to significant morbidity due to the capillary leak syndrome that affects the heart, lungs, kidneys, and central nervous system, leading to oliguria, ischemia, and confusion. ${ }^{14}$ Although clinicians are now better able to prevent and manage the side effects associated with IL-2, its toxicity has prompted a search for predictive markers to determine which patients would benefit from this therapy. Retrospective studies have shown that patients with a good performance status (Eastern Cooperative Oncology Group status 0-1), a normal lactate dehydrogenase level, fewer than three organs involved, and cutaneous or subcutaneous metastases have the highest probability of achieving a durable complete response to high-dose IL-2. ${ }^{15}$ Additionally, retrospective analysis has suggested that patients with NRAS-mutated melanoma may have longer overall survival after IL-2 therapy compared with those having tumors lacking the NRAS mutation. ${ }^{16} \mathrm{~A}$ retrospective study of the response of patients with metastatic melanoma to high-dose IL-2 therapy demonstrated that tumors expressing an immune signature by the DNAmediated annealing, selection, and ligation technique are more likely to respond. ${ }^{17}$ This gene expression profiling demonstrated that patients with genes involved in the innate immune response, including various chemokines, cytokines, and cytokine receptors, along with growth factors related to melanoma, are more likely to respond to IL-2 therapy. ${ }^{17}$ Analysis of absolute lymphocyte count has shown no correlation with progression-free survival in patients with metastatic melanoma receiving high-dose IL-2. ${ }^{18}$

Serum vascular endothelial growth factor and fibronectin levels at baseline are negative predictors of response to IL-2, ${ }^{19}$ whereas patients who develop hypothyroidism during therapy with IL-2 are more likely to respond. ${ }^{20}$ These observations have furthered interest in prospectively validating predictive markers in studies such as the IL-2 SELECT trial (ClinicalTrials.gov identifier NCT01288963).

Development of clinically useful predictive biomarkers to better select patients who will benefit from cytokine therapy is essential. Although cytokine therapy has severe toxicities 
that limit its clinical use, it has a place in the management of advanced melanoma since there are low but measurable overall response and durable remission rates.

\section{Immunotherapy through checkpoint blockade}

There is now a better understanding of the immune system and its role in tumor biology. The immune system's innate checkpoints and regulatory pathways limit the naturally occurring immune responses against tumors. Targeted agents are being developed with the goal of strengthening a weak immune response to tumor cells through blocking these checkpoint pathways, resulting in an immune-mediated antitumor effect. CTLA-4, a key negative regulator in one checkpoint pathway, is a molecule that is expressed on the surface of active T-lymphocytes and suppresses the ability of the immune system to respond to the tumor. Blocking the CTLA-4 antigen with an antibody leads to an antitumor response (Figure 1). ${ }^{20}$

Agents such as ipilimumab (Bristol-Myers Squibb Co, Princeton, NJ, USA), which is a fully human monoclonal anti-CTLA-4 antibody, have shown an overall response rate of $11.1 \%$ in a Phase II study. ${ }^{21}$ One-year and 2-year survival rates were $47.2 \%$ and $32.8 \%$, respectively, with a median overall survival of 10.2 months in patients with previously treated, unresectable stage III/IV melanoma. ${ }^{22}$ The FDA approved ipilimumab for the treatment of metastatic melanoma based on the double-blind Phase III MDX010-20 study in 676 patients with previously treated metastatic melanoma who were randomized $3: 1: 1$ to receive ipilimumab $3 \mathrm{mg} / \mathrm{kg}$ plus a peptide vaccine (gp100), ipilimumab $3 \mathrm{mg} / \mathrm{kg}$ alone, or the peptide vaccine alone. ${ }^{23}$ Median overall survival was 10.0 months and 10.1 months in the arms containing ipilimumab compared with 6.4 months in the arm containing the peptide vaccine alone (hazard ratio $0.68 ; P<0.003$ ). The 24 -month overall survival in the ipilimumab plus gp100 group was $21.6 \%$ versus $23.5 \%$ in the ipilimumab alone group and $13.7 \%$ in the gp100 alone group. ${ }^{24}$ Patients achieved prolonged survival with the use of ipilimumab.

Combination therapy of ipilimumab $10 \mathrm{mg} / \mathrm{kg}$ plus dacarbazine versus dacarbazine alone also demonstrated significantly prolonged overall survival; the group receiving ipilimumab plus dacarbazine had an overall survival of 11.2 months versus 9.1 months in the dacarbazine alone group. ${ }^{25}$ The 3-year overall survival in the ipilimumab group was $20.8 \%$ versus $12.2 \%$ in the dacarbazine alone group. Although there is an overall survival benefit from combination therapy of dacarbazine and ipilimumab, this combination regimen has not been widely adopted in clinical practice due to higher toxicity. Approximately $56.3 \%$ of patients treated with ipilimumab plus dacarbazine experienced grade $3 / 4$ adverse events. ${ }^{25}$ It is possible that the higher levels of toxicity with this combination may be due to the higher dosing of ipilimumab at $10 \mathrm{mg} / \mathrm{kg}$ rather than the lower dosing seen in the Phase III MDX010-20 study with ipilimumab $3 \mathrm{mg} / \mathrm{kg}$. Survival analyses of four Phase II trials using ipilimumab at $3 \mathrm{mg} / \mathrm{kg}$ or $10 \mathrm{mg} / \mathrm{kg}$ demonstrated a proportion of patients $(12.3 \%-49.5 \%)$ who remained alive 5 years after starting treatment. ${ }^{26}$ Currently, a prospective randomized trial comparing ipilimumab $3 \mathrm{mg} / \mathrm{kg}$ versus $10 \mathrm{mg} / \mathrm{kg}$ in patients with metastatic melanoma to determine the optimal single-agent dose is being conducted (ClinicalTrials.gov identifier NCT01515189).

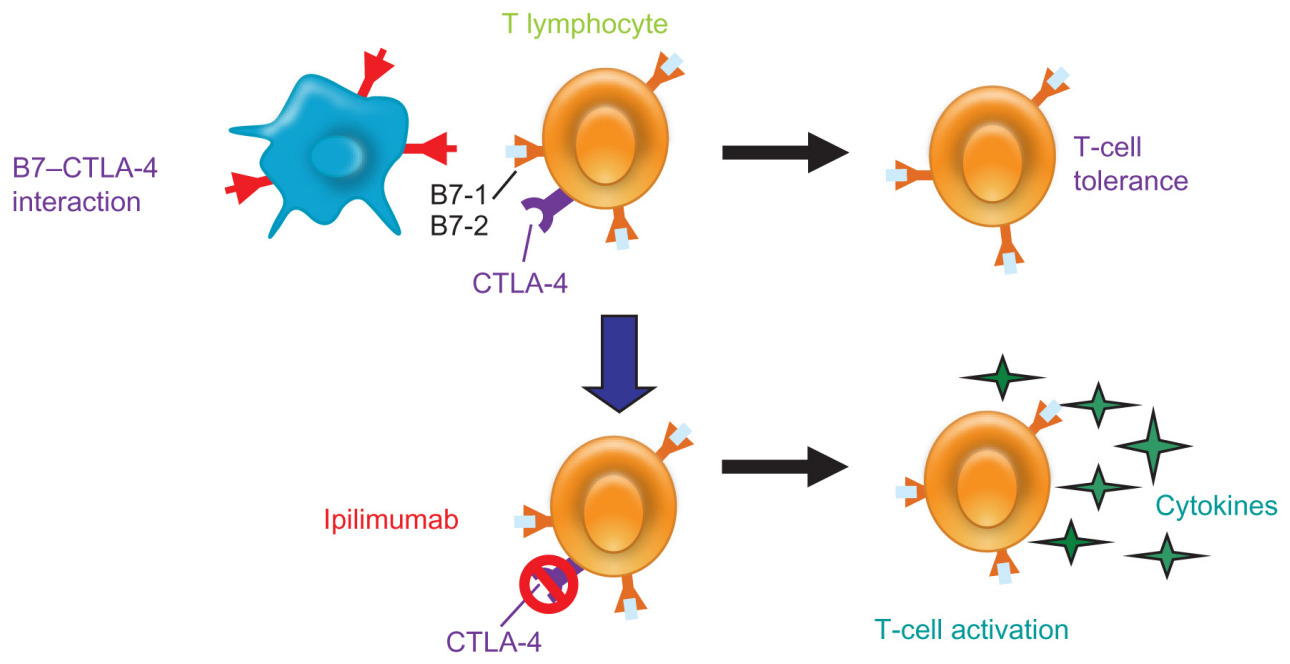

Figure I CTLA-4 pathway is a key regulator of T-cell activation and tolerance. CTLA-4 is a negative regulator that is expressed on the surface of T-lymphocytes and suppresses the immune system. Blocking CTLA-4 with ipilimumab, an anti-CTLA-4 antibody, allows for an antitumor response. Abbreviation: CTLA-4, Cytotoxic T-Lymphocyte Antigen 4. 
Response with ipilimumab has also been seen in patients with asymptomatic metastatic lesions in the brain. ${ }^{27}$ Further, retreating patients with ipilimumab who had progressed after initially achieving disease control with ipilimumab has demonstrated some antitumor activity and clinical benefit, with only mild to moderate toxicities noted at the time of retreatment. ${ }^{28}$

Targeted immunotherapy agents such as ipilimumab are associated with toxicity profiles that are thought to be immune-related. These events include colitis/diarrhea, hepatitis, endocrinopathies, nephritis, uveitis, and inflammatory myopathy. ${ }^{29}$ One of the most notable toxicities is severe diarrhea (grade 3 in approximately $8 \%$ of patients). This colitis is thought to be secondary, due to dysregulation of the gastrointestinal mucosal immunity, leading to alterations in the enteric flora and infiltration of inflammatory cells in the gastrointestinal mucosa. ${ }^{30}$ Ipilimumab is also associated with grade 3 fatigue and an increase in serum aspartate transaminase. ${ }^{27}$ Although the incidence of autoimmune hypophysitis is low (1.8\%), this can lead to life-threatening adrenal insufficiency if not recognized early. ${ }^{31}$ There have also been a few cases of irreversible hypopituitarism requiring life-long hormonal replacement therapy. ${ }^{31}$ Whether immune-related adverse events correlate with treatment response has not been conclusively demonstrated for any of these events. ${ }^{23}$

Most immune-related adverse events are manageable and reversible with corticosteroids. ${ }^{22} \mathrm{~A}$ risk evaluation and mitigation strategy, published and made available online as per FDA requirements for ipilimumab-related severe immune-mediated adverse reactions, delineates how to dose reduce ipilimumab and administer corticosteroids in a safe manner. ${ }^{32}$

With the advent of targeted immunotherapy, there is a need to reassess the traditional means of measuring response to therapy with the Response Evaluation Criteria in Solid Tumors (RECIST) criteria. ${ }^{33}$ In a Phase II clinical trial with ipilimumab, four distinct response patterns were associated with favorable survival: shrinkage of baseline lesions without new lesions, durable stable disease, response after an increase in total tumor burden, and response in the presence of new lesions. ${ }^{33}$ The transient lesion growth with the use of effective immunotherapy is likely due to immune cells infiltrating the lesion, which leads to the appearance of tumor growth. ${ }^{34}$ Furthermore, standard endpoints through RECIST assess antitumor response to cytotoxic agents. However, targeted immunotherapy is not cytotoxic but rather cytostatic, so more time may elapse before clinical effects are quantifiable. ${ }^{34} \mathrm{As}$ a result, novel criteria that consider the nature of clinical benefit from immunotherapy, known as immune-related response criteria, have been proposed as alternatives to RECIST and utilized in clinical trials of ipilimumab. ${ }^{33}$ Additionally, other endpoints such as overall survival may be more useful in studies of targeted immunotherapy. ${ }^{34}$

Ipilimumab has demonstrated clinical benefit in advanced melanoma. However, ongoing research seeks to identify which patients would benefit most from anti-CTLA-4 therapy. Studies have correlated an increase in absolute lymphocyte count in the patient's peripheral blood to $1,000 \mu \mathrm{L}$ after two ipilimumab treatments with significantly improved clinical benefit, ie, a median overall survival of 11.9 months compared with 1.4 months in patients with an absolute lymphocyte count less than $1,000 \mu \mathrm{L}$ after two treatments. ${ }^{35}$ A correlation between increase in mean absolute lymphocyte count while on therapy and greater clinical benefit has been reported. ${ }^{36}$ In a small prospective biomarker study, high pretreatment tumor expression of forkhead box P3, a transcription factor that regulates the development and function of regulatory T-cells, was associated with increased clinical activity in patients receiving ipilimumab. ${ }^{37}$ This study also showed that while on therapy, changes in the microenvironment of the tumor with an increase in tumor-infiltrating lymphocytes was associated with improved response to ipilimumab. ${ }^{37}$ Further research is in progress to clarify which patients should be treated with drugs like ipilimumab.

\section{Molecularly targeted therapy and its contribution to tumor immunology}

The development of novel agents is due to the identification of driver mutations such as the BRAF mutation, which is found in approximately $40 \%-50 \%$ of melanoma tumors and is involved in tumor evasion of the immune system. ${ }^{38}$ Patients with BRAF-mutant primary melanoma have worse outcomes and are often diagnosed younger than patients with wild-type BRAF disease ${ }^{39}$ The FDA approved the use of vemurafenib (Genentech USA, Inc., South San Francisco, CA, USA), a small-molecule BRAF kinase inhibitor, based on the Phase III BRIM3 study of 675 patients with previously untreated, BRAF V600E mutation-positive, unresectable, or metastatic melanoma. In this study, the risk of death was reduced by $56 \%$ for patients who received vemurafenib compared with those who received chemotherapy $(P<0.0001) .{ }^{40}$ Additionally, the median progression-free survival was 5.3 months for those who received vemurafenib compared with 1.6 months for those who received chemotherapy. A retrospective study showed that vemurafenib did not reduce the incidence of brain metastases ${ }^{41}$ In the Phase I and II studies of vemurafenib, 
$38 \%$ of patients required dose reduction of the drug because of toxic effects, the most common being rash, fatigue, arthralgia, alopecia, photosensitivity, nausea, and diarrhea. ${ }^{40}$ Of note, vemurafenib is associated with unique cutaneous toxicities, specifically photosensitivity or eruptive squamous cell carcinoma. Rarely, patients may also develop keratosis pilaris-like eruptions, seborrheic dermatitis-like rashes, and hyperkeratotic tender plantar papules. ${ }^{42}$ Vemurafenib blocks the mitogen-activated protein kinase pathway, resulting in increased expression of melanocyte differentiation antigens (Figure 2). These antigens are recognized by antigen-specific T-lymphocytes and lead to an antitumor immune response. ${ }^{43}$ Currently, clinical trials are evaluating combination regimens of vemurafenib and other targeted therapies that can block other oncogenic drivers. These combinations are discussed in the section "Combination Therapies".

\section{Novel targeted immunotherapy agents}

Other novel treatments directing the immune system to activate T-cells against cancer cells have been studied. The PD-1 pathway and its role in the development of various malignancies is, thus far, the most interesting (Figure 3). PD-L1, also called B7-H1, is a B7 family molecule that is expressed in several types of cancer. The corresponding receptor, PD-1, is expressed on activated T-cells. ${ }^{44}$ The PD-1/PD-L1 pathway impedes the $\mathrm{CD} 8^{+}$cytotoxic T-lymphocytes to recognize and destroy tumor cells and is thought to be exploited by tumors to promote tumor survival by evading the host's native antitumor immune responses. ${ }^{45}$ Blocking PD-L1 or PD-1 using monoclonal antibodies is able to reverse this resistance (Figures 4 and 5). ${ }^{45}$ The PD-1 receptor also interacts with PD-L2, a ligand that is expressed on solid tumors and antigenpresenting cells such as activated macrophages and dendritic cells. ${ }^{46}$ Because PD-L2 interacts with PD-1 in a different way than PD-L1 does, it has been suggested that the two ligands may have nonoverlapping roles in the PD-1 pathway. ${ }^{46}$ The relative advantages of targeting the complete pathway (antiPD-1) or the individual ligands (anti-PD-L1 or anti-PD-L2) for therapeutic purposes are not completely understood.

A Phase I study of single-agent nivolumab (formerly MDX-1106 or BMS-936558, Bristol-Myers Squibb Co), a fully human IgG4 PD-1 blocking antibody, in refractory solid tumors demonstrated tumor responses in patients with melanoma. ${ }^{47} \mathrm{~A}$ similar Phase I study conducted by Brahmer et al demonstrated that an antibody against PD-1 induces antitumor activity in patients with advanced malignancy, including melanoma. ${ }^{48}$ Patients receiving nivolumab showed a median overall survival of 16.8 months, with $40 \%$ of patients alive at 3 years. ${ }^{49}$ These encouraging data have prompted further trials. Currently, there is an ongoing Phase III trial of nivolumab (anti-PD-1) versus dacarbazine in patients with metastatic melanoma (ClinicalTrials. gov identifier NCT01721772). Another anti-PD-1 monoclonal antibody, lambrolizumab (Merck Sharp \& Dohme Corp, Whitehouse Station, NJ, USA), has shown antitumor activity with a manageable side effect profile in a Phase I trial of patients with advanced melanoma.$^{50}$ Common side effects associated with the PD-1 antibody are grade 1/2 fatigue, diarrhea, xerostomia, and

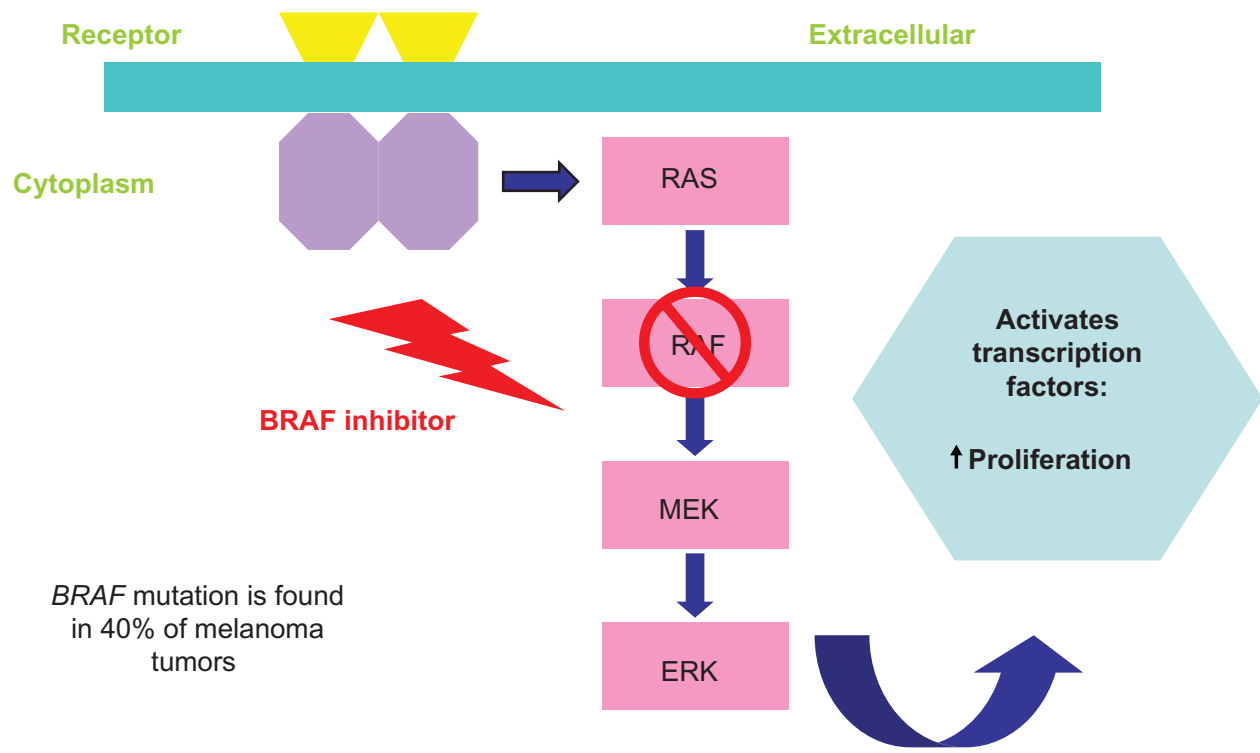

Figure 2 BRAF inhibitor pathway. BRAF inhibitors block the mitogen-activated protein kinase pathway thus increasing melanocyte antigen presentation, which is recognized by T-lymphocytes and leads to increased antitumor response by activating transcription factors and increasing proliferation of lymphocytes. 


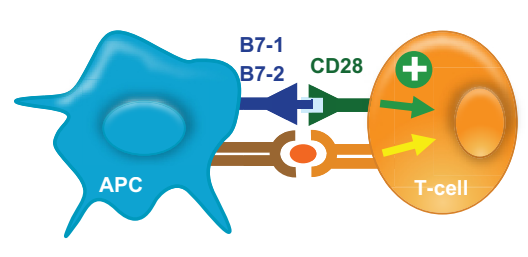

T-cell priming

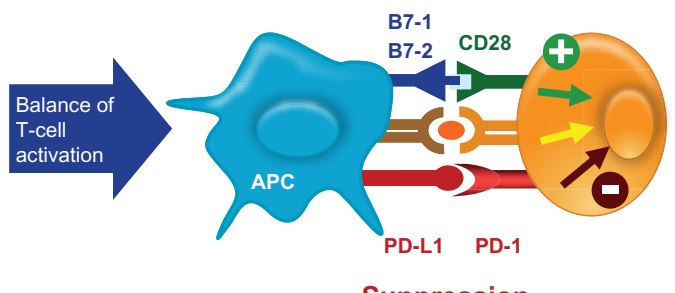

Suppression

(anergy, exhaustion, death)

Figure 3 PD-I/PD-LI immune checkpoint pathway. PD-LI is expressed on antigen-presenting cells whereas PD-I is expressed on T-lymphocytes. The PD-I-PD-LI pathway impairs the ability of T-lymphocytes to mount an immune response. This is a key checkpoint in a normal immune response.

Abbreviation: APC, antigen-presenting cells.

pruritus. ${ }^{51}$ The PD-L1 antibody has shown objective responses in Phase I trials in nine of 52 patients with melanoma. ${ }^{52}$ Nine percent of patients in the Phase I study had grade 3/4 toxicities, with adrenal insufficiency or infusion-related toxicity. ${ }^{52}$ MPDL3280A (Roche, Basel, Switzerland), an engineered PD-L1 antibody, showed an overall response rate of $26 \%$ with a 24 -week progression-free survival of $35 \%$ in locally advanced or metastatic melanoma. ${ }^{53}$ Grade $3 / 4$ toxicities included development of hyperglycemia (7\%), elevated alanine transaminase $(7 \%)$, and elevated aspartate transaminase $(4 \%){ }^{53}$

Initial Phase I studies suggested that the level of PD-L1 expression on tumor cells correlated with response to treatment with PD-1 antibody. ${ }^{38}$ Topalian et al noted that of the 17 patients in the Phase I study who had PD-L1-negative tumors, none had an objective response to treatment with anti-PD-1 ${ }^{54}$ Subsequent studies have demonstrated that, regardless of the PD-L1 status of the tumor, there is an antitumor response in patients treated with the PD-1 antibody. Patients whose melanoma was found to be PD-L1 positive had an overall response rate of $44 \%$ compared with tumors that were PD-L1 negative, which had an overall response rate of $17 \% .{ }^{55}$ Grosso et al demonstrated that although there were higher rates of response noted in PD-L1-positive tumors, there was still some clinical benefit in tumors that were PD-L1-negative. ${ }^{55}$ Furthermore, 20\% of PD-L1-negative tumors had a response to treatment when treated with MPDL3280A, a PD-L1 antibody. ${ }^{53}$ It is unclear why PD-L1-negative tumors would respond to blockade of the PD-1 pathway. There is speculation that the current means to determine the PD-L1 status of a tumor is not yet precise.

\section{Combination therapies}

Development of these agents has improved the treatment of advanced melanoma, and their diverse mechanisms of action support the rationale for combination regimens. The intent of combining therapies is to improve efficacy while maintaining a manageable level of toxicity. Immunotherapy has demonstrated the ability to maintain durable remissions in a low percentage of patients, whereas targeted therapies are able to block an oncogenic driver and induce faster responses. Thus, there is a rationale behind combining these different modalities. ${ }^{56}$ Studies evaluating combination immunotherapy have had mixed results. A prospective Phase III trial randomizing patients with advanced melanoma to chemotherapy composed of tamoxifen, cisplatin, and dacarbazine or this same regimen followed by IFN and IL-2 therapy demonstrated increased toxicity with no survival benefit. ${ }^{18}$

\section{Ipilimumab combinations}

In a small trial performed at the US National Cancer Institute, the combination of IL-2 and ipilimumab has showed a median overall survival of 16 months with a 5 -year survival of $25 \% .{ }^{57}$ Nonrandomized data combining
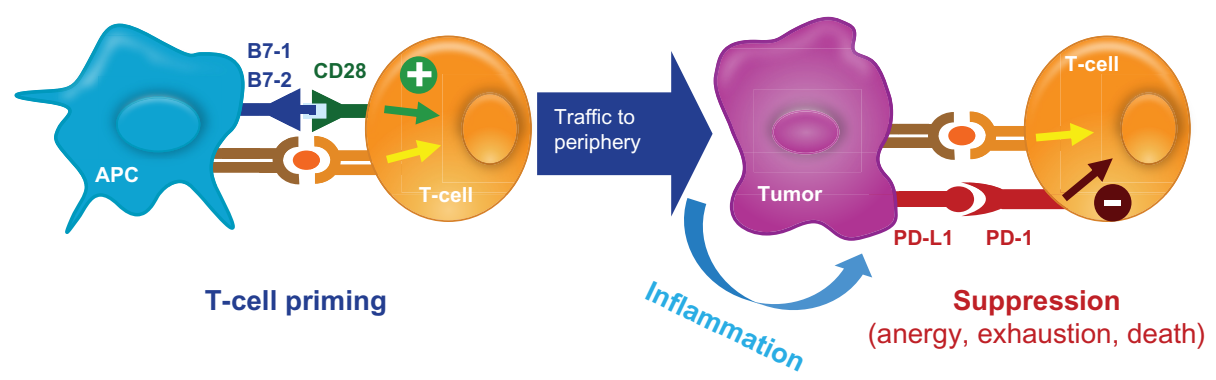

Figure 4 PD-LI expressed on tumor cells endogenously or induced by association with T-cells. Tumor cells express PD-LI and bind to PD-I on T-lymphocytes, thus suppressing the ability of T-lymphocytes to recognize and destroy tumor cells. This mechanism of evading the innate immune system is exploited by various types of tumor cells. Abbreviation: APC, antigen-presenting cells. 


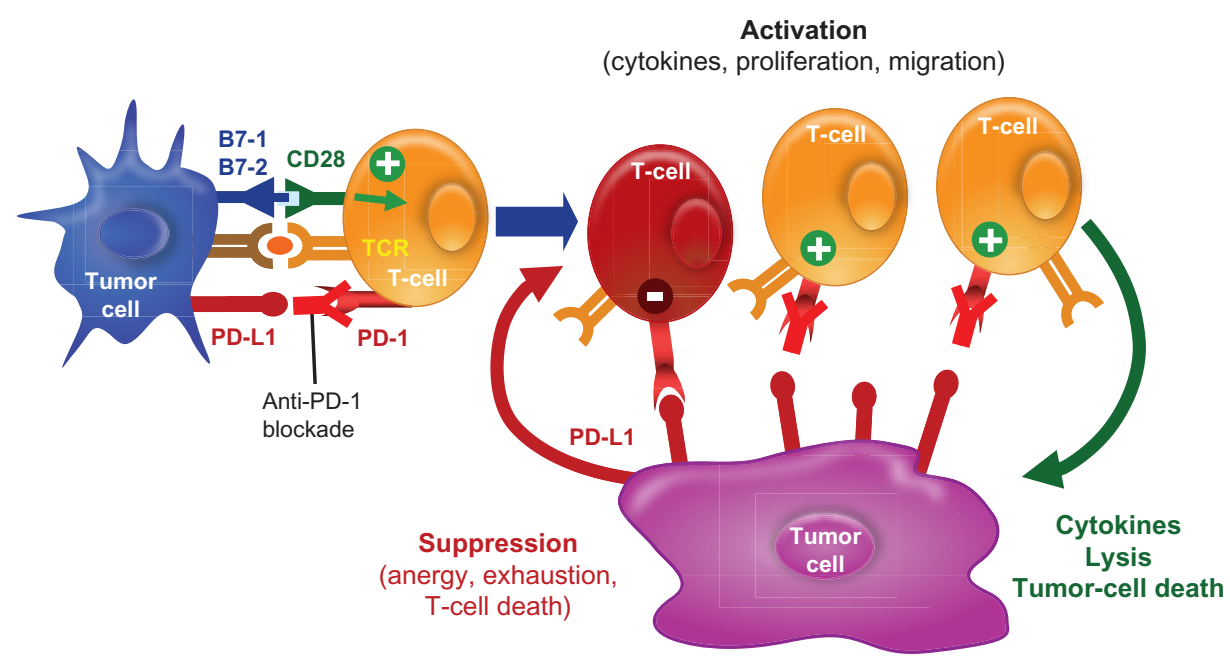

Figure 5 Anti-PD-I blocks T-cell suppression. Blocking the PD-I-PD-LI pathway by using either a PD-I antibody or a PD-LI antibody allows for T-lymphocytes to mount a robust immune response against tumor cells.

Abbreviation: TCR, T-cell receptor.

ipilimumab and bevacizumab (Genentech USA, Inc.) revealed a clinical benefit in 14 of 21 patients, suggesting that there may be an additive effect. ${ }^{58}$ This is being further evaluated in a Phase II study (ClinicalTrials.gov identifier NCT01950390). A Phase Ib study of ipilimumab with peginterferon alfa-2b (H Lee Moffitt Cancer Center and Research Institute, Tampa, FL, USA, and Merck Sharp \& Dohme Corp, ClinicalTrials.gov identifier NCT01496807) in patients with unresectable melanoma demonstrated an excellent response rate with tolerable side effects, thus warranting additional studies evaluating this combination. ${ }^{59}$ A recent Phase I study highlighted the safety concerns of concurrent administration of vemurafenib and ipilimumab $(3 \mathrm{mg} / \mathrm{kg}$ ) with dose-limiting toxicities of grade 3 transaminase elevations. ${ }^{60}$ Although both drugs have different mechanisms of action, further investigation is needed on how to administer these drugs safely in combination.

Another Phase I clinical trial is studying the effects of CTLA-4 blockade with ipilimumab in conjunction with PD-1 blockade through nivolumab to enhance the immune system's response to melanoma. ${ }^{61}$ Clinical activity for concurrent therapy is superior to monotherapy of either drug with both deep and rapid tumor response rates, with more than $80 \%$ tumor reduction at 12 weeks in $30 \%$ of patients. ${ }^{61}$ The related adverse events for concurrent therapy were higher in frequency compared with monotherapy of either drug; however, these toxicities were manageable with immunosuppressants. ${ }^{61}$

Combination of ipilimumab with granulocytemacrophage colony-stimulating factor (Sanofi-Aventis US LLC, Bridgewater, NJ, USA), which augments dendritic cell activity and enhances the antitumor effects of T-cells and B-cells, has shown a significant increase in overall survival, with one-year survival of $67.9 \%$ versus $51.2 \%$ in the monotherapy arm. ${ }^{62}$ There were no significant notable differences in toxicity between the two arms of therapy. ${ }^{62}$

In addition, combination of ipilimumab and radiation therapy to elicit the abscopal effect has been described in a patient with metastatic melanoma. ${ }^{23}$ This phenomenon is mediated by activation of the immune system and local radiotherapy and leads to regression of metastatic cancer at a distance from the irradiated site.

An ongoing Phase II study of nivolumab (anti-PD-1) given sequentially with ipilimumab in patients with advanced or metastatic melanoma will guide clinicians about the best sequence to administer these novel agents in patients with advanced melanoma (ClinicalTrials.gov identifier NCT01783938).

\section{Vemurafenib combinations}

Understanding the mechanism behind acquiring resistance to vemurafenib therapy has helped identify potential therapeutic targets that function downstream from BRAF in this oncogenic pathway. This has led to research into combination therapy with BRAF and MEK inhibitors. ${ }^{63}$ In a Phase I study evaluating patients with metastatic melanoma and BRAF V600 mutations, patients were randomly assigned to receive combination therapy with dabrafenib (a BRAF inhibitor; GlaxoSmithKline, Research Triangle Park, NC, USA) plus trametinib (MEK-inh) (GlaxoSmithKline) compared with dabrafenib alone.$^{64}$ Complete or partial responses were seen in $76 \%$ of the combination therapy group versus $54 \%$ in the dabrafenib alone group. The combination regimen was safely 
tolerated at full monotherapy doses, with pyrexia being more common in the combination group than in the monotherapy group (71\% versus 26\%). Currently, a Phase III study is being conducted to assess the response to dabrafenib plus trametinib versus vemurafenib alone in patients with metastatic melanoma and the BRAF V600 mutation (ClinicalTrials.gov identifier NCT01597908). A trial of dabrafenib with trametinib is being conducted to assess whether resistance to BRAF inhibition can be prevented. ${ }^{65}$ Preliminary data from this Phase I/II trial suggest that dual blockade may delay clinical resistance to BRAF inhibition. ${ }^{65}$ Future clinical research may include trials to study the combination of BRAF inhibitors together with immunotherapy.

\section{Best algorithms}

With the rapid emergence of efficacious agents for advanced melanoma, investigators have attempted to devise algorithms using these novel agents in advanced melanoma. Prolonged overall survival can be seen in a certain group of patients treated with immunotherapy first, such as IL-2 prior to vemurafenib, with overall survival of 31.2 months and response rates of $75 \% .{ }^{66}$ Conclusions from this retrospective analysis were that response rates to vemurafenib following immunotherapy were equivalent to those seen in previously untreated patients. However, progression-free and overall survival rates were poor in patients who received ipilimumab after vemurafenib. This was most likely due to rapid progression of disease at the time of discontinuing vemurafenib. ${ }^{66}$

A retrospective study analyzing the use of vemurafenib followed by ipilimumab and vice versa in 34 BRAF mutation-positive patients suggested that some patients are at a high risk of rapid disease progression once relapsed with a BRAF inhibitor. ${ }^{67}$ These patients may not have time to complete ipilimumab therapy if the BRAF inhibitor is administered first. Thus, BRAF mutation-positive patients may benefit from administration of ipilimumab first followed by a BRAF inhibitor.

Upcoming studies are being considered by various cooperative groups to develop clinical trials that would assess what sequence of therapy would be the best in patients with advanced melanoma. Additional factors, such as declining performance status, tumor-related symptoms, and disease growth rate as measured by imaging studies or lactate dehydrogenase levels should be taken into account when clinicians are deciding which therapy should be initiated first. A proposed algorithm for patients with metastatic melanoma harboring the BRAF V600E mutation is described in Figure 6.

\section{Proposed management algorithm}

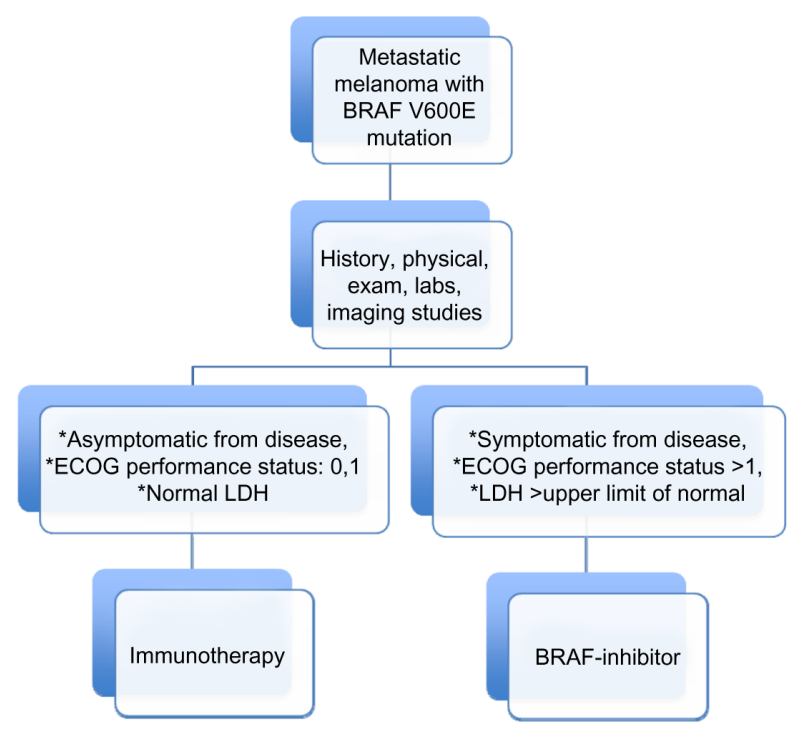

Figure 6 Patients with metastatic melanoma harboring the BRAF V600E mutation who have symptomatic disease, elevated LDH levels, and poor performance status should be offered initial therapy with BRAF inhibitors. Conversely, patients who are asymptomatic from their disease and have normal LDH and good performance status may derive more benefit from immunotherapeutic agents first.

Abbreviations: ECOG, Eastern Cooperative Oncology Group; LDH, lactate dehydrogenase.

\section{Future directions}

Future research is aimed at using immune checkpoint inhibitors or other targeted agents such as BRAF inhibitors in the adjuvant setting. Conducting randomized controlled trials of various combinations of newly approved drugs and clarifying the best way to sequence these agents is necessary. Additionally, ongoing research is leading to a better understanding of pathways such as PD-1/PDL-1 that regulate and affect the immune response against tumors. As molecular identification of tumor antigens continues, there may be different subsets of tumors that activate different signaling pathways and thus downregulate expression of immunoregulatory genes. ${ }^{68}$ Pathways currently under investigation include Notch signaling, Stat 3 phosphorylation, $\beta$-catenin stabilization, ErbB4, c-met, and phosphatidylinositide-3 kinase. Studying these pathways will hopefully lead to development of small-molecule inhibitors that can improve immune-mediated regulatory effects against the tumor.

Tremendous progress has been made in the management of melanoma with targeted forms of immunotherapy by improving clinical efficacy, reducing toxicities, combining therapies, selecting for patients who will derive benefits from certain treatments, and devising new algorithms in sequentially administering these novel agents. Immunotherapy has 
a therapeutic role not only in melanoma, but also in other tumor types, such as renal cell carcinoma, non-small-cell lung cancer, gastric cancer, and prostate cancer. These novel targeted immunotherapies have substantial positive effects on the outcome for patients with advanced melanoma, with improvements in overall survival, tolerable side effects, and durable benefits off treatment. It is imperative that clinicians learn about these new therapeutics; they undoubtedly will become essential components of the treatment of malignant melanoma and of other malignancies as well.

\section{Disclosure}

DMD has participated on advisory boards for Genentech, Bristol-Myers Squibb, Merck, and Prometheus Labs. NS reports no conflicts of interest in this work. The authors take full responsibility for the content of this publication and confirm that it reflects their viewpoint and medical expertise. The authors also wish to acknowledge StemScientific, funded by Bristol-Myers Squibb, for providing editorial support. Neither Bristol-Myers Squibb nor StemScientific influenced the content of the manuscript, nor did the authors receive financial compensation for authoring the manuscript.

\section{References}

1. Jemal A, Siegel R, Xu J, Ward E. Cancer statistics, 2010. CA Cancer J Clin. 2010;60(5):277-300.

2. Reed KB, Brewer JD, Lohse CM, Bringe KE, Pruitt CN, Gibson LE. Increasing incidence of melanoma among young adults: an epidemiologic study in Olmsted County, Minnesota. Mayo Clin Proc. 2012;87(4): 328-334.

3. Guy GP, Ekwueme DU. Years of potential life lost and indirect costs of melanoma and non-melanoma skin cancer: a systematic review of the literature. Pharmacoeconomics. 2011;29(10):863-874.

4. Balch CM, Gershenwald JE, Soong SJ, et al. Final version of 2009 AJCC melanoma staging and classification. J Clin Oncol. 2009;27(36): 6199-6206.

5. Legha SS. The role of interferon alfa in the treatment of metastatic melanoma. Semin Oncol. 1997;24(1 Suppl 4):S24-S31.

6. Mocellin S, Pasquali S, Rossi CR, Nitti D. Interferon alpha adjuvant therapy in patients with high-risk melanoma: a systematic review and meta-analysis. J Natl Cancer Inst. 2010;102(7):493-501.

7. Kirkwood JM, Strawderman MH, Ernstoff MS, Smith TJ, Borden EC, Blum RH. Interferon alfa- $2 b$ adjuvant therapy of high risk resected cutaneous melanoma: the Eastern Cooperative Oncology Group Trial EST 1684. J Clin Oncol. 1996;14(1):7-17.

8. Jonasch E, Kumar UN, Linette GP, et al. Adjuvant high-dose interferon alfa-2b in patients with high-risk melanoma. Cancer J. 2000;6(3): $139-145$.

9. McMasters KM, Edwards MJ, Ross MI, et al. Ulceration as a predictive marker for response to adjuvant interferon therapy in melanoma. Ann Surg. 2010;252(3):460-465.

10. Eggermont A, Suciu S, Testori A, et al. Ulceration and stage are predictive of interferon efficacy in melanoma: results of the phase III adjuvant trials EORTC 18952 and EORTC 18991. Eur J Cancer. 2012;48(2): 218-225.

11. Gogas H, Ioannovich J, Dafni U, et al. Prognostic significance of autoimmunity during treatment of melanoma with interferon. $N$ Engl J Med 2006;354(7):709-718.
12. Sullivan RJ, Flaherty KT. BRAF in melanoma: pathogenesis, diagnosis, inhibition, and resistance. J Skin Cancer. 2011;2011:423239.

13. Atkins MB, Lotze MT, Dutcher JP, et al. High-dose recombinant interleukin 2 therapy for patients with metastatic melanoma: analysis of 270 patients treated between 1985 and 1993. J Clin Oncol. 1999;17(7):2105-2116.

14. Schwartz RN, Stover L, Dutcher J. Managing toxicities of high-dose interleukin-2. Oncology (Williston Park). 2002;16(11 Suppl 13): 11-20.

15. Petrella T, Quirt I, Verma S, et al. Single-agent interleukin-2 in the treatment of metastatic melanoma. Curr Oncol. 2007;14(1):21-26.

16. Joseph RW, Sullivan RJ, Harrell R, et al. Correlation of NRAS mutations with clinical response to high-dose IL-2 in patients with advanced melanoma. J Immunother. 2012;35(1):66-72.

17. Sullivan RJ, Hoshida Y, Brunet J, et al. A single center experience with high-dose (HD) IL-2 treatment for patients with advanced melanoma and pilot investigation of a novel gene expression signature as a predictor of response. J Clin Oncol. 2009;27(Suppl 15):Abstr 9003.

18. Rosenberg S, Yang J, Schwartzentruber D, et al. Prospective randomized trial of the treatment of patients with metastatic melanoma using chemotherapy with cisplatin, dacarbazine, and tamoxifen alone or in combination with interleukin-2 and interferon alfa-2b. J Clin Oncol. 1999;17(3):968-975.

19. Sabatino M, Kim-Schulze S, Panelli MC, et al. Serum vascular endothelial growth factor and fibronectin predict clinical response to high-dose interleukin-2 therapy. J Clin Oncol. 2009;27(16):2645-2652.

20. Atkins MB, Mier JW, Parkinson DR, Gould JA, Berkman EM, Kaplan MM. Hypothyroidism after treatment with interleukin-2 and lymphokineactivated killer cells. $N$ Engl J Med. 1988;318(24):1557-1563.

21. Wolchok JD, Neyns B, Linette G, et al. Ipilimumab monotherapy in patients with pretreated advanced melanoma: a randomised, double-blind, multicentre, phase 2, dose-ranging study. Lancet Oncol. 2010;11(2):155-164.

22. O'Day SJ, Maio M, Chiarion-Sileni V, et al. Efficacy and safety of ipilimumab monotherapy in patients with pretreated advanced melanoma: a multicenter single-arm phase II study. Ann Oncol. 2010;21(8): $1712-1717$.

23. Weber JS, Kähler KC, Hauschild A. Management of immune-related adverse events and kinetics of response with ipilimumab. J Clin Oncol. 2012;30(21):2691-2697.

24. Hodi FS, O'Day SJ, McDermott DF, et al. Improved survival with ipilimumab in patients with metastatic melanoma. $N$ Engl $J$ Med. 2010;363(8):711-723.

25. Robert C, Thomas L, Bondarenko I, et al. Ipilimumab plus dacarbazine for previously untreated metastatic melanoma. $N$ Engl $J$ Med. 2011;365(26):2517-2526.

26. Lebbe C, Weber J, Maio B, et al. Long-term survival in patients with metastatic melanoma who received ipilimumab in four phase II trials. J Clin Oncol. 2013;31(Suppl 15):Abstr 9053.

27. Margolin K, Ernstoff MS, Hamid O, et al. Ipilimumab in patients with melanoma and brain metastases: an open-label, phase 2 trial. Lancet Oncol. 2012;13(5):459-465.

28. Robert C, Schadendorf D, Messina M, Hodi FS, O'Day S; MDX010-20 Investigators. Efficacy and safety of retreatment with ipilimumab in patients with pretreated advanced melanoma who progressed after initially achieving disease control. Clin Cancer Res. 2013;19(8): 2232-2239.

29. Di Giacomo AM, Biagioli M, Maio M. The emerging toxicity profiles of anti-CTLA-4 antibodies across clinical indications. Semin Oncol. 2010;37(5):499-507.

30. Berman D, Parker SM, Siegel J, et al. Blockade of cytotoxic T-lymphocyte antigen-4 by ipilimumab results in dysregulation of gastrointestinal immunity in patients with advanced melanoma. Cancer Immun. 2010;10:11.

31. Torino F, Barnabei A, De Vecchis L, Salvatori R, Corsello SM. Hypophysitis induced by monoclonal antibodies to cytotoxic $\mathrm{T}$ lymphocyte antigen 4: challenges from a new cause of a rare disease. Oncologist. 2012;17(4):525-535. 
32. US Food and Drug Administration. Yervoy (ipilimumab): risk evaluation and mitigation strategy (REMS) - severe immune-mediated adverse reactions. 2011. Available from: http://www.fda.gov/Safety/ MedWatch/SafetyInformation/SafetyAlertsforHumanMedicalProducts/ ucm249770.htm. Accessed February 21, 2013.

33. Wolchok JD, Hoos A, O'Day S, et al. Guidelines for the evaluation of immune therapy activity in solid tumors: immune-related response criteria. Clin Cancer Res. 2009;15(23):7412-7420.

34. Hales RK, Banchereau J, Ribas A, et al. Assessing oncologic benefit in clinical trials of immunotherapy agents. Ann Oncol. 2010;21(10):1944-1951.

35. Ku GY, Yuan J, Page DB, et al. Single-institution experience with ipilimumab in advanced melanoma patients in the compassionate use setting: lymphocyte count after 2 doses correlates with survival. Cancer. 2010;116(7):1767-1775.

36. Berman DM, Wolchok J, Weber J, et al. Association of peripheral blood absolute lymphocyte count (ALC) and clinical activity in patients (pts) with advanced melanoma treated with ipilimumab. J Clin Oncol. 2009;27(Suppl 15):Abstr 3020.

37. Hamid O, Chasalow SD, Tsuchihashi Z, et al. Association of baseline and on-study tumor biopsy markers with clinical activity in patients (pts) with advanced melanoma treated with ipilimumab. J Clin Oncol. 2009;27(Suppl 15):Abstr 9008.

38. Rubinstein JC, Sznol M, Pavlick AC, et al. Incidence of the V600K mutation among melanoma patients with BRAF mutations, and potential therapeutic response to the specific BRAF inhibitor PLX4032. JTransl Med. 2010;8:67.

39. Brissy S, Gaudy-Marqueste C, Mallet S, et al. BRAF mutation as a pejorative marker in metastatic melanoma. J Clin Oncol. 2012;30 Suppl:Abstr 8555.

40. Chapman PB, Hauschild A, Robert C, et al; for the BRIM-3 Study Group. Improved survival with vemurafenib in melanoma with BRAF V600E mutation. $N$ Engl J Med. 2011;364(26):2507-2516.

41. Gummadi T, Dronca RS, Kim C, et al. Impact of BRAF mutation and effectiveness of BRAF inhibitor on the brain metastases in patients with metastatic melanoma. J Clin Oncol. 2013;31:15 Suppl: Abstr 9048.

42. Larkin JM, Queirolo P, Arance AM, et al. An open-label, multicenter safety study of vemurafenib (PLX4032, RO5185426) in patients with metastatic melanoma. J Clin Oncol. 2012;30 Suppl:Abstr 8517.

43. Topalian SL, Hodi FS, Brahmer JR, et al. Safety, activity, and immune correlates of anti-PD-1 antibody in cancer. $N$ Engl J Med. 2012;366(26):2443-2454.

44. Keir ME, Butte MJ, Freeman GJ, Sharpe AH. PD-1 and its ligands in tolerance and immunity. Annu Rev Immunol. 2008;26:677-704.

45. Hirano F, Kaneko K, Tamura H, et al. Blockade of B7-H1 and PD-1 by monoclonal antibodies potentiates cancer therapeutic immunity. Cancer Res. 2005;65(3):1089-1096.

46. Ghiotto M, Gauthier L, Serriari N, et al. PD-L1 and PD-L2 differ in their molecular mechanisms of interaction with PD-1. Int Immunol. 2010;22(8):651-660.

47. Patnaik A, Kang SP, Tolcher AW, et al. Phase I study of MK-3475 (antiPD-1 monoclonal antibody) in patients with advanced solid tumors. J Clin Oncol. 2012;30 Suppl:Abstr 2512.

48. Brahmer JR, Drake CG, Wollner I, et al. Phase I study of singleagent anti-programmed death-1 (MDX-1106) in refractory solid tumors: safety, clinical activity, pharmacodynamics, and immunologic correlates. J Clin Oncol. 2010;28(19):3167-3175.

49. Sznol M, Kluger H, Hodi F, et al. Survival and long-term follow up of safety and response in patients (pts) with advanced melanoma (MEL) in a phase I trial of nivolumab (anti-Pd-1; BMS-936558; ONO-4538). J Clin Oncol. 2013;31(Suppl 15):Abstr 9006.

50. Ribas A, Robert C, Daud A, et al. Clinical efficacy and safety of lambrolizumab (MK-3475, Anti-PD-1 monoclonal antibody) in patients with advanced melanoma. J Clin Oncol. 2013;31(Suppl 15): Abstr 9010
51. Business Wire. Merck presents early-stage interim data for MK-3475, an investigational therapy for the treatment of advanced melanoma phase II study being initiated [press release]. Whitehouse Station, NJ, USA: November 11, 2012. Available from: http://www.businesswire. com/news/home/20121111005025/en/Merck-Presents-Early-StageInterim-Data-MK-3475-Investigational\#.U3FPcvmSxho. Accessed May 13, 2014.

52. Sznol M, Powderly JD, Smith DC, et al. Safety and antitumor activity of biweekly MDX-1106 (Anti-PD-1, BMS-936558/ONO-4538) in patients with advanced refractory malignancies. $J$ Clin Oncol. 2010;28(Suppl 15):Abstr 2506.

53. Hamid O, Sosman JA, Lawrence DP, et al. Clinical activity, safety, and biomarkers of MPDL3280A, an engineered PD-L1 antibody in patients with locally advanced or metastatic melanoma. J Clin Oncol. 2013;31(Suppl 15):Abstr 9010.

54. Topalian SL, Hodi FS, Brahmer JR, et al. Safety, activity, and immune correlates of anti-PD-1 antibody in cancer. $N$ Engl J Med. 2012;366(26):2443-2454.

55. Grosso J, Horak C, Inzunza D, et al. Association of tumor PD-L1 expression and immune biomarkers with clinical activity in patients (pts) with advanced solid tumors treated with nivolumab (anti-PD1; BMS-936558; ONO-4538). J Clin Oncol. 2013;31(Suppl 15):Abstr 3016.

56. Ribas A, Hersey P, Middleton MR, et al. New challenges in endpoints for drug development in advanced melanoma. Clin Cancer Res. 2012;18(2):336-341.

57. Postow MA, Callahan MK, Barker CA, et al. Immunologic correlates of the abscopal effect in a patient with melanoma. $N$ Engl J Med. 2012;366(10):925-931.

58. Prieto PA, Yang JC, Sherry RM, et al. CTLA-4 blockade with ipilimumab: long-term follow-up of 177 patients with metastatic melanoma. Clin Cancer Res. 2012;18(7):2039-2047.

59. Kuchadkar RR, Gibney GT, Weber J, et al. A phase IB study of ipilimumab with peginterferon alfa-2b in patients with unresectable melanoma. J Clin Oncol. 2013;31(Suppl 15):Abstr 9079.

60. Ribas A, Hodi S, Callahan M, et al. Hepatotoxicity with combination of vemurafenib and ipilimumab. N Engl J Med. 2013;368(14): 1365-1366.

61. Wolchok J, Kluger H, Callahan M. Safety and clinical activity of nivolumab (anti-PD-1, BMS-936558, ONO-4538) in combination with ipilimumab in patients with advanced melanoma. J Clin Oncol. 2013;31(Suppl 15):Abstr 9012.

62. Hodi FS, Lee SJ, McDermott DF. Multicenter, randomized phase II trial of GM-CSF (GM) plus ipilimumab (Ipi) versus Ipi alone in metastatic melanoma: E1608. J Clin Oncol. 2013;31(Suppl 15):Abstr CRA9007.

63. Garber K. Beyond ipilimumab: new approaches target the immunological synapse. J Natl Cancer Inst. 2011;103(14):1079-1082.

64. Infante JR, Falchook GS, Lawrence DP, et al. Phase I/II study to assess safety, pharmacokinetics, and efficacy of the oral MEK $1 / 2$ inhibitor GSK1120212 (GSK212) dosed in combination with the oral BRAF inhibitor GSK2118436 (GSK436). J Clin Oncol. 2011;29 Suppl: Abstr 8503.

65. Sosman J, Daud A, Weber J, et al. BRAF inhibitor (BRAFi) dabrafenib in combination with the MEK1/2 inhibitor (MEKi) trametinib in BRAFinaïve and BRAFi-resistant patients with BRAF mutation-positive metastatic melanoma. J Clin Oncol. 2013; 31(Suppl 15):Abstr 9005.

66. Ackerman A, McDermott DF, Lawrence LP, et al. Outcomes of patients with malignant melanoma treated with immunotherapy prior to or after vemurafenib. J Clin Oncol. 2012;30 Suppl:Abstr 8569.

67. Ascierto PA, Simeone E, Giannarelli D, Grimaldi AM, Romano A, Mozzillo N. Sequencing of BRAF inhibitors and ipilimumab in patients with metastatic melanoma: a possible algorithm for clinical use. J Transl Med. 2012;10:107.

68. Gajewski TF, Fuertes M, Spaapen R, Zheng Y, Kline J. Molecular profiling to identify relevant immune resistance mechanisms in the tumor microenvironment. Curr Opin Immunol. 2011;23(2): 286-292. 


\section{Publish your work in this journal}

Cancer Management and Research is an international, peer-reviewed open access journal focusing on cancer research and the optimal use of preventative and integrated treatment interventions to achieve improved outcomes, enhanced survival and quality of life for the cancer patient The journal welcomes original research, clinical \& epidemiological studies, reviews \& evaluations, guidelines, expert opinion \& commentary, case reports \& extended reports. The manuscript management system is completely online and includes a very quick and fair peerreview system, which is all easy to use. Visit http://www.dovepress.com/ testimonials.php to read real quotes from published authors.

Submit your manuscript here: http://www.dovepress.com/cancer-management-and-research-journal 